Case Report

\title{
Co-existence of gastric adenocarcinoma and neuroendocrine carcinoma: a rare entity
}

\author{
Mainali Nirajan, ${ }^{1}$ Nepal Niraj, ${ }_{1}^{1}$ Choudhary Prabesh kumar, ${ }^{1}$ Sinha Amrita, ${ }^{1}$ \\ Rajbanshi Saroj, ${ }^{2}$ Rauniyar Shyam, ${ }^{3}$ Yadav Ajay ${ }^{4}$
}

${ }^{1}$ Department of pathology, Nobel medical college teaching hospital, Biratnagar, Nepal.

${ }^{2}$ Department of surgery, Golden hospital, Biratnagar, Nepal.

${ }^{3}$ Department of medicine, Golden hospital, Biratnagar, Nepal.

${ }^{4}$ Department of radiology and imaging, Golden hospital, Biratnagar, Nepal.

\section{Keywords: \\ Adenocarcinoma; \\ Carcinoma; \\ Collision; \\ Neuroendocrine}

\begin{abstract}
A mixed adenoneuroendocrine carcinoma is a tumor composed of both adenocarcinoma and neuroendocrine carcinoma components, with each comprising at least one-third of the lesion, as defined by the World Health Organization classification of neuroendocrine neoplasms in 2010.. A 67-years-old male was admitted to the hospital with symptoms suggesting gastric cancer. Histopathology examination from endoscopic biopsy revealed adenocarcinoma. Later partial gastrectomy specimen examination the lesion show presence of well differentiated adenocarcinoma along with neuro endocrine carcinoma.
\end{abstract}

Correspondence:

Dr. Nirajan Mainali, MBBS, MD

Assistant Professor, Pathology

Nobel Medical College Teaching Hospital

Orcid ID: 0000-0002-6648-1914

Email: mainali_nirajan@hotmail.com

Received : February 26th, 2017 ; Accepted : June 2nd, 2017; Published : September 1, 2017

Citation: Mainali N, Nepal N, Choudhary PK, Sinha A, et al. Co-existence of gastric adenocarcinoma

and neuroendocrine carcinoma: a rare entity. J Pathol Nep. 2017;7:1121-3. doi: 10.3126/jpn.

v7i2.18030

Copyright: This is an open-access article distributed under the terms of the Creative Commons Attribution 4.0 International License, which permits unrestricted use, distribution, and reproduction in any medium, provided the original author and source are credited.

\section{INTRODUCTION}

A mixed adenoneuroendocrine carcinoma is a tumor composed of both adenocarcinoma and neuroendocrine carcinoma (NEC) components, with each comprising at least one-third of the lesion, as defined by the World Health Organization (WHO) classification of neuroendocrine neoplasms in 2010. ${ }^{1}$ Before 2010, this tumor was reported as a mixed or composite tumor. In general, neuroendocrine carcinoma is considered to grow rapidly and have a poor prognosis, ${ }^{3}$ whereas mixed adenoneuroendocrine carcinoma tumors grow based on the component with greater malignant potential. ${ }^{4}$ Most neuroendocrine carcinomas, including mixed adenoneuroendocrine carcinoma lesions, are diagnosed at an advanced stage and mainly treated with surgical resection or chemotherapy. Diagnosis is mainly based on the tumor architecture, being completed by the immunostains with specific neuroendocrine markers 


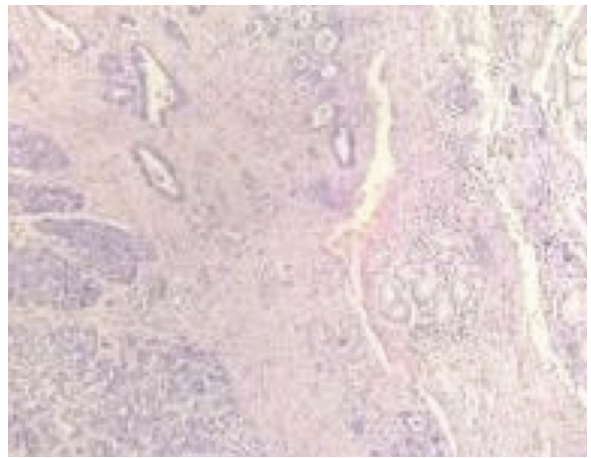

Figure 1: Photomicrograph showing normal gastric mucosa adenocarcinoma and neuroendocrine carcinoma (HE stain; X 50).

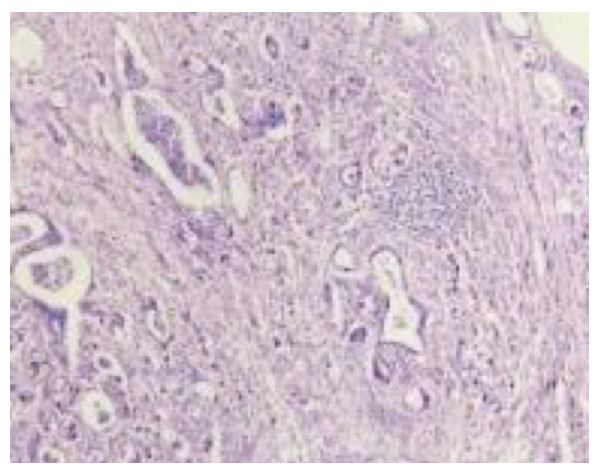

Figure 3: Photomicrograph showing tumor cells arranged in glandular pattern (HE stain, X40).

such as chromogranin, synaptophysin, CD56, and neuronspecific enolase (NSE), combined with the markers on non-endocrine differentiation such as keratin 7 (for gastric tumors) and Keratin 20, CDX2, and carcinoembryonic antigen (CEA), respectively, for colorectal segments. ${ }^{5}$

\section{CASE REPORT}

A 67-years-old male was admitted to the hospital with symptoms suggesting gastric cancer: weight loss, hematemesis and melena. Ultrasonography revealed a hypoechoic mass in the submucosal layer. On upper gastrointestinal endoscopy, ulcerating lesion was found in the antral region. Biopsy was taken was sent for histopathological examination in the department for histopathology. Microscopy examination reveals adenocarcinoma. Partial gastrectomy was done and the specimen was sent for histopathological examination. $3.5 \mathrm{~cm}$ sub epithelial grey white mass was found 2 $\mathrm{cm}$ away from proximal resected margin with normal looking mucosa. Seven lymph nodes were dissected out. On histopathological examination the lesion shows presence of well differentiated adenocarcinoma along with neuroendocrine carcinoma (fig.1). Tumor cells with various shape and size glands were seen with cells having high N/C ratio, hyperchromatic nuclei and occasional visible nucleoli without mucinous component. Proliferations of small round

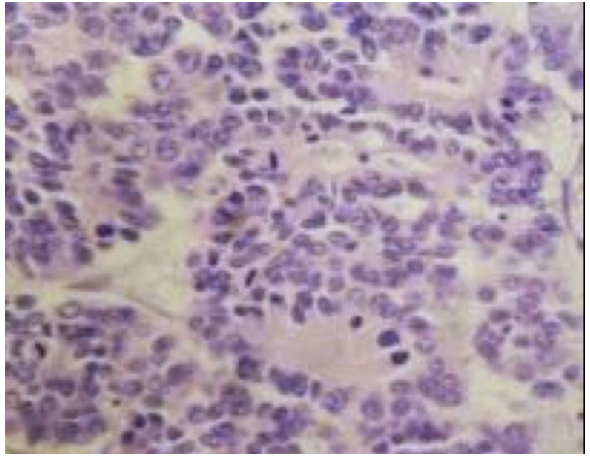

Figure 2: Photomicrogrpah showing rosettes and mitotic figures in neuroendocrine carcinoma (HE stain; X100).

cells with stippled chromatin with rosette formation were also seen (fig.2). Other area shows malignant cells arranged in well and poorly formed glandular pattern. (fig. 3) Both of the components covered about half of the dissected tumor part. Seven lymph nodes were dissected out, out of which three showed metastatic deposits of both entities. Perineural and blood vessel invasion were not noted. Immunohistochemistry could not be done due to its unavailability in the department.

\section{DISCUSSION}

A new classification system of digestive neuroendocrine tumors (NETs) was formulated and presented in the 2010 revision of the WHO classification of digestive tumors. This system recognizes three main categories according to the Ki-67 index: NET G1, NET G2 and NEC. The submucosal component in the present case corresponded to NEC. The original definition of mixed endocrine-exocrine carcinoma (MEEC) was incorporated into the WHO classification of gastroenteropancreatic endocrine tumors in 2000, which states that an MEEC is a tumor with two different cell populations, each of which composes at least one-third of the tumor area. Thereafter, the new WHO classification proposed the term MANEC to classify this type of tumor based on similar criteria. ${ }^{1}$ In the present case, each of the NEC and adenocarcinoma components accounted for nearly half of the tumor; thus, the lesion was consistent with the definition of MANEC. The carcinogenesis of MANEC is unclear $^{6}$ but the role of unusual intestinal metaplasia and hypergastrinemia needs to be under consideration. ${ }^{7}$ Previous reports have noted the coexistence of gastric NEC with a scant amount of adenocarcinoma tissue. Nishikura et al. noted that $70.6 \%$ of gastric NEC cases include an adenocarcinoma component in the mucosa and/or submucosa. ${ }^{8}$ In the present case, the endoscopic findings showed a reddish area of erosion, which corresponded to the adenocarcinoma component, and endoscopic ultrasonography revealed a hypoechoic mass in the submucosal layer, which reflected the NEC component. When the tumor was resected, the $\mathrm{NEC}$ and adenocarcinoma components were each found to 
occupy approximately half of the tumor. Three out of seven lymph nodes show metastatic deposits of both the entity in our case where as in the study done by gurzu et al $22 / 32$ lymph node showed deposits in the excised lymph node. ${ }^{5}$

\section{Conflict of Interest: None}

\section{REFERENCES}

1. Bosman FT CF, Hruban RH, Theise ND. Tumors of the digestive system.World Health Organization Classification of Tumours. Pathology and genetics. Lyon: IARC Press;2010. 64-8 p.

2. Borch K, Ahren B, Ahlman H, Falkmer S, Granerus G, Grimelius L. Gastric carcinoids: biologic behavior and prognosis after differentiated treatment in relation to type. Annals of surgery. 2005;242:64-73. Crossref

3. Domori K, Nishikura K, Ajioka Y, Aoyagi Y. Mucin phenotype expression of gastric neuroendocrine neoplasms: analysis of histopathology and carcinogenesis. Gastric cancer 2014;17:263-72. Crossref
4. Gurzu S, Kadar Z, Bara T, et al. Mixed adenoneuroendocrine carcinoma of gastrointestinal tract: report of two cases. World J Gastroenterol.2015;21:1329-33. Crossref

5. Fukuba N, Yuki T, Ishihara S, Sonoyama H, Tada Y, Kusunoki R, et al. Gastric mixed adenoneuroendocrine carcinoma with a good prognosis. Internal medicine.2014;53:2585-8. Crossref

6. Yamauchi H, Sakurai $\mathrm{S}$, Nakazawa $\mathrm{N}$, et al. A case of mixed adenoneuroendocrine carcinoma of the stomach with focal intestinal metaplasia and hypergastrinemia. International surgery. 2015;100:5627. Crossref

7. Nishikura K, Watanabe H, Iwafuchi M, Fujiwara T, Kojima K, Ajioka Y. Carcinogenesis of gastric endocrine cell carcinoma: analysis of histopathology and p53 gene alteration. Gastric cancer. 2003;6:203-9. Crossref 